\title{
Structural Insights into the Active Site Formation of DUSP22 in N-loop-containing Protein Tyrosine Phosphatases
}

\author{
Chih-Hsuan Lai ${ }^{1}{ }^{\mathbb{C}}$, Co-Chih Chang ${ }^{1}$, Huai-Chia Chuang ${ }^{2}$, Tse-Hua Tan ${ }^{2} \mathbb{D}$ \\ and Ping-Chiang Lyu 1,*(D) \\ 1 Institute of Bioinformatics and Structural Biology, Department of Life Science, National Tsing Hua \\ University, Hsinchu 30013, Taiwan; chsuan.1@gmail.com (C.-H.L.); cochihchang@gmail.com (C.-C.C.) \\ 2 Immunology Research Center, National Health Research Institutes, Zhunan 35053, Taiwan; \\ cinth@nhri.org.tw (H.-C.C.); ttan@nhri.org.tw (T.-H.T.) \\ * Correspondence: pclyu@mx.nthu.edu.tw; Tel.: +886-3574-2762; Fax: +886-3571-5934
}

Received: 28 August 2020; Accepted: 9 October 2020; Published: 12 October 2020

\begin{abstract}
Cysteine-based protein tyrosine phosphatases (Cys-based PTPs) perform dephosphorylation to regulate signaling pathways in cellular responses. The hydrogen bonding network in their active site plays an important conformational role and supports the phosphatase activity. Nearly half of dual-specificity phosphatases (DUSPs) use three conserved residues, including aspartate in the D-loop, serine in the P-loop, and asparagine in the N-loop, to form the hydrogen bonding network, the D-, $\mathrm{P}-, \mathrm{N}$-triloop interaction (DPN-triloop interaction). In this study, DUSP22 is used to investigate the importance of the DPN-triloop interaction in active site formation. Alanine mutations and somatic mutations of the conserved residues, D57, S93, and N128 substantially decrease catalytic efficiency $\left(k_{\text {cat }} / K_{\mathrm{M}}\right)$ by more than $10^{2}$-fold. Structural studies by NMR and crystallography reveal that each residue can perturb the three loops and induce conformational changes, indicating that the hydrogen bonding network aligns the residues in the correct positions for substrate interaction and catalysis. Studying the DPN-triloop interaction reveals the mechanism maintaining phosphatase activity in $\mathrm{N}$-loop-containing PTPs and provides a foundation for further investigation of active site formation in different members of this protein class.
\end{abstract}

Keywords: hydrogen bonding network; active site; N-loop; DUSP22; DUSPs; Cys-based PTPs

\section{Introduction}

Cysteine-based protein tyrosine phosphatases (Cys-based PTPs) are important enzymes that remove phosphate from phosphorylated residues and regulate several signaling pathways in cellular processes, including cell differentiation, proliferation, and immune response [1-3]. The somatic mutations on Cys-based PTPs can disrupt cellular processes and induce high risk of human diseases [4-6]. Cys-based PTPs consist of two major subfamilies: classical PTPs and dual-specificity phosphatases (DUSPs) [2]. The phosphatase domains in classical PTPs and DUSPs are formed by twisted $\beta$-sheets surrounded by $\alpha$-helices. The active site is a crevice on the surface of the structure. The PTP signature motif, the P-loop $\left(\mathrm{CX}_{5} \mathrm{R}\right)$, is located at the bottom of this crevice, and it provides the binding site as well as a conserved cysteine to trigger catalytic activation [1,2]. The loops surrounding the P-loop play important roles in supporting catalytic reactions. The D-loop (WPD-loop) provides a conserved aspartate to act as a general acid/base during the catalytic reaction, and the Q-loop provides the conserved glutamines to stabilize water molecules for hydrolysis of the cysteinyl-phosphate intermediate and correct positioning of the active site residues [1,7-9]. The active site, which comprises 
the D-loop, P-loop, and Q-loop, is highly conserved in classical PTPs, and the mechanism is well understood. However, the Q-loop sequence varies in DUSPs [1]. Several members of DUSPs replace the Q-loop with an N-loop, which has a conserved sequence of PNXXF, in contrast to the Q-loop motif (QXXXQ) (Figure 1A) [10]. The first crystal structure of DUSPs is DUSP3, published by Saper's group in 1996 [11], while the N-loop is described by Johnson's group in 2012 [10]. The active site, which consists of the D-loop, P-loop, and N-loop, is present in $46 \%$ of DUSPs but has not been clearly investigated. These two groups of PTPs include more than half of the members in Cys-based PTPs; Q-loop-containing PTPs (classical PTPs) constitute 32\% of the 116 members in Cys-based PTPs $[2,12]$ while N-loop-containing PTPs constitute $25 \%$. Since the two groups are clearly divided in phylogenetic relationships [13], investigation of N-loop-containing PTPs can provide information for further understanding the relationship between the structure and catalytic activity in the Cys-based PTP family.

(A)





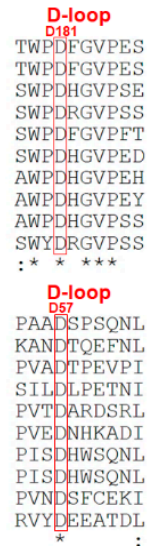

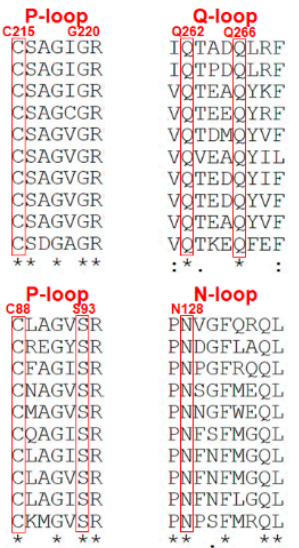

(B) PTP1B



(C)DUSP22

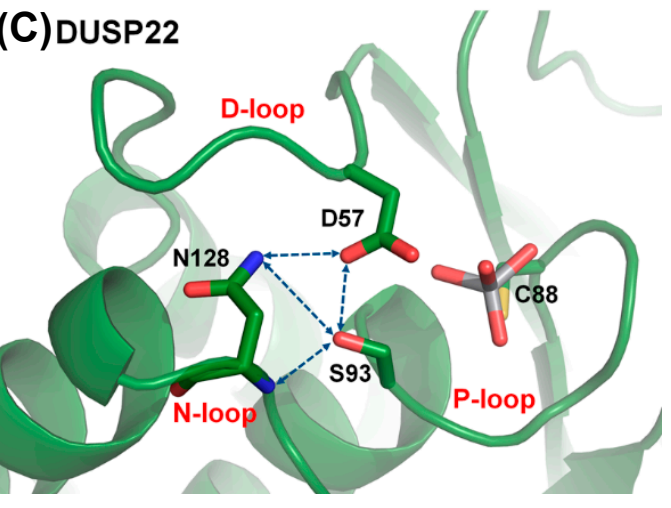

Figure 1. The D-, P-, N-triloop (DPN-triloop) interaction in N-loop-containing protein tyrosine phosphatases (PTPs). (A) The sequence alignment showed the difference between Q-loop-containing PTPs and N-loop-containing PTPs. N-loop-containing PTPs had an N-loop motif (PNXXF), which was different from the Q-loop motif (QXXXQ). The key residues (red) in PTP1B and DUSP22 are labeled above the sequence alignment. (B) In the structure of PTP1B (PDB ID: 3I80), structural water (red ball) can form the hydrogen bonding with the side chain of D181, the side chain of Q262, the side chain of Q266, the backbone amide of F182, the backbone amide of R221, and the oxygens of the ligand in active site. This water-mediated DPQ-triloop interaction connected the D-loop, the P-loop, the Q-loop, and the ligand, and aligned these residues to the correct position. (C) In the structure of DUSP22 (PDB ID: 6LVQ), the active site contained a hydrogen bonding network named the DPN-triloop interaction, which was formed by three residues: D57 in the D-loop, S93 in the P-loop, and N128 in the N-loop. The side chains of D57, S93, and N128 formed hydrogen bonds with each other, and the backbone amide of N128 formed hydrogen bonding with the side chain of S93. Hydrogen bonding is displayed as blue dotted lines, and the residues as well as the ligand, $\mathrm{VO}_{4}$, are shown by sticks. 
PTP1B (PTPN1) is a well-known model for studying the reaction cycle in Q-loop-containing PTPs. The reaction cycle consists of two steps and three loops that participate in the reaction: the D-loop, P-loop, and Q-loop (Figure S1) [1,2]. In the first step, the P-loop provides arginine to stabilize substrate binding, and nucleophilic cysteine attacks the phosphorus atom. At this time, catalytic aspartate (D181) in the D-loop acts as a general acid to donate a proton for substrate dissociation and results in a cysteinyl-phosphate intermediate. In the second step, conserved glutamine (Q262) in the Q-loop motif rotates toward the ligand and acts as an anchor to stabilize the position of water for catalytic aspartate $[7,8]$. Catalytic aspartate in the second step acts as a general base that accepts protons from water and results in nucleophilic water, facilitating hydrolysis of the cysteinyl-phosphate intermediate and releasing phosphate [1,7]. The catalytic reaction is probably similar between Q-loop-containing PTPs and N-loop-containing PTPs because all of them have the P-loop and D-loop [1], but the different sequences in the Q-loop motif and N-loop motif suggest that they might have different roles in active site formation.

More differences between the two groups can be found in the active site and the selected ligands. The Q-loop-containing PTPs have a deep active site pocket with a depth of $\sim 9 \AA$, and the substrate is phosphotyrosine (pTyr). The N-loop-containing PTPs have a shallow active site pocket of $\sim 6 \AA$, and the substrates include pTyr as well as pThr/pSer [1,2,11,14]. In the Q-loop-containing PTPs, the active site contains a structural water molecule that interacts with a catalytic aspartate in the D-loop and the conserved glutamines in the Q-loop [7,9]. This structural water molecule (W1) is highly conserved in the crystal structures of Q-loop-containing PTPs [7], and interacts with the D-loop, P-loop, Q-loop, and ligand that align the residues in the active site (Figure 1B) [8,9]. In contrast, the catalytic aspartate in the D-loop is exposed on the surface of N-loop-containing PTPs and interacts with a serine in the P-loop and an asparagine in the N-loop through a hydrogen bonding network [10]. The interaction between the serine and aspartate in N-loop-containing PTPs replaces the position of structural water in Q-loop-containing PTPs [11], and the hydrogen bonds contributed by the serine and asparagine are described to be involved in maintaining the catalytic aspartate in a catalytically favorable conformation [10], but the mechanism has not been clarified. To investigate the role of this hydrogen bonding network, somatic mutations of serine and asparagine are searched in cancer database, and DUSP22 includes both S93 and N128 mutations in the P-loop and N-loop (Tables S1 and S2) $[15,16]$.

DUSP22 is a small phosphatase containing 184 residues, and the sequence contains a catalytic domain as well as an N-terminal myristoylation site $[17,18]$. This enzyme is broadly expressed in different tissues and plays important roles in the regulation of signaling pathways, such as inactivating the $\mathrm{T}$ cell receptor (TCR) signaling pathway, activating the JNK signaling pathway, and regulating the phosphorylation of focal adhesion kinase (FAK) [17,19-22]. DUSP22-deficient animal models exhibit risks of autoimmunity, and downregulation of DUSP22 in patients exhibits a strong relationship with systemic lupus erythematosus (SLE) and inflammatory bowel disease (IBD) $[19,23,24]$. The phosphatase domain of DUSP2 2 consists of five twisted $\beta$-sheets and six $\alpha$-helices, and the structural scaffold is similar to that of DUSP3 (VHR) [17], while DUSP22 does not contain an N-terminal $\alpha$-helix surrounded by the N-loop. The P-loop is located between $\beta 5$ and $\alpha 3$ and forms a shallow active site with a catalytic aspartate, D57, at a depth of $4.5 \AA$ [17]. In this region, the catalytic activity is facilitated by C 88 in the P-loop and D57 in the D-loop [17], and the hydrogen bonding network is formed by D57 in the D-loop, S93 in the P-loop, and N128 in the N-loop. We mutated these residues (D57, S93, and N128) to study whether the DPN-triloop interaction is associated with catalytic activity as well as structural formation and reveals a crucial role that is probably applicable to all N-loop-containing PTPs. 


\section{Results}

\subsection{Characteristics of the DPN-triloop Interaction}

The hydrogen bonding network in N-loop-containing PTPs contains three conserved residues, namely, aspartate in the D-loop, serine in the P-loop, and asparagine in the N-loop (Figure 1A). The sequence alignment indicated that these residues were highly conserved in N-loop-containing PTPs, which included three families: atypical DUSPs, MKPs, and Slingshots (Figure 1A). The members included 26 phosphatases and 3 pseudophosphatases (DUSP27, STYX, and STYXL1) (Figure S2), suggesting that the DPN-triloop interaction not only played a role in catalysis but also participated in structural formation. In DUSP22, the hydrogen bonding network was formed by the side chain of D57 in the D-loop, S93 in the P-loop, and N128 in the N-loop. DUSP22 not only contains the hydrogen bonds which formed with the side chain of D57 but also includes the hydrogen bonds formed between S93 and N128 through the side chains and backbone amide (Figure 1C). This hydrogen bonding network was described as the DPN-triloop interaction in this study, and it probably contributed to stabilizing the three loops similar to the water-mediated DPQ-triloop interaction in Q-loop-containing PTPs (Figure 1B). To confirm that the DPN-triloop interaction was important for forming the structure of the active site, we mutated D57, S93, and N128 to detect phosphatase activity.

\subsection{The Role of DPN-triloop Interaction in Phosphatase Activity}

The residues, D57, S93, and N128, were mutated to study their effect on catalytic activity via a $p$-nitrophenyl phosphate (pNPP) kinetic assay. D57 is a catalytic aspartate [17], and the role of catalytic aspartate has been verified by alanine and asparagine mutations in previous studies [25,26]. S93 and N128 sites had unknown effects. We referred to the somatic mutated residues that were equivalent to S93 and N128 in different N-loop-containing PTPs in the cancer databases and found several reports, including S285L in DUSP4, S298G in DUSP6, S249F in DUSP16, S155F in DUSP19, S111N in DUSP21, N297S in DUSP2, N125D in DUSP15, N160D in STYX, N168K in DUPD1, and N453K in SSH3 (Table S1) [15,16]. To investigate the roles of these residues and the effects of somatic mutations, S93 and N128 were replaced with alanine and the referred mutants (Tables S1 and S2). N128D was selected due to similar side chain and enough protein yield, and S93N was the only S93 site mutant that induced enough protein yield.

The kinetic parameters of DUSP22 and the mutations in the DPN-triloop interaction are listed in Table 1 . The enzyme kinetic of wild-type (WT) DUSP22 showed that the $K_{\mathrm{M}}$ was $0.64 \mathrm{mM}$ and the $k_{\text {cat }}$ was $0.65 \mathrm{~s}^{-1}$, which were similar to previous published results [27]. The mutations of D57 decreased $k_{\text {cat }}$ by 26-31 times, while the $K_{\mathrm{M}}$ values were different between D57N and D57A. The $K_{\mathrm{M}}$ of D57N was similar to that of the WT, but the $K_{M}$ of D57A was increased more than 8 times. The mutations of $S 93$ and N128 increased $K_{\mathrm{M}}$ and decreased $k_{\text {cat }}$ as well. S93A and S93N increased the $K_{\mathrm{M}}$ by $14-19$ times, and the $k_{\text {cat }}$ declined more than 10 times. N128A and N128D increased the $K_{\mathrm{M}}$ more than 30 times, but the $k_{\text {cat }}$ values were different. N128D decreased the $k_{\text {cat }}$ by 16 times, while the $k_{\text {cat }}$ of N128A only decreased 2.5 times. The catalytic efficiency $\left(k_{\text {cat }} / K_{\mathrm{M}}\right)$ indicated that the mutations on D57, S93, and N128 decreased 40-500 times. These results reflected important information indicating that the mutations affected both substrate affinity and catalytic activity and were associated with disruption of the DPN-triloop interaction. The primary difference between D57N and the other mutations was the $K_{\mathrm{M}}$ value. The asparagine of D57N probably maintained the DPN-triloop interaction due to the similar side chain, but the other mutations did not maintain the interaction. The alanine mutations in D57, S93, and N128, might interfere with the formation of the DPN-triloop interaction, and S93N and N128D might disrupt the interaction through longer side chain and charge repulsion. Importantly, both mutations of S93 and N128 decreased the catalytic efficiency $\left(k_{\text {cat }} / K_{\mathrm{M}}\right)$ by more than $10^{2}$ times, revealing that S93 and N128 were necessary for maintaining phosphatase activity. The detailed structural studies of the DPN-triloop interaction were further investigated by NMR spectroscopy and protein crystallography. 
Table 1. Kinetic parameters of the mutations in the DPN-triloop interaction.

\begin{tabular}{cccc}
\hline & $\boldsymbol{K}_{\mathbf{M}}(\mathbf{m M})$ & $\boldsymbol{k}_{\text {cat }}\left(\mathbf{s}^{\mathbf{- 1}}\right)$ & $\boldsymbol{k}_{\text {cat }} / \boldsymbol{K}_{\mathbf{M}}\left(\mathbf{s}^{\left.-\mathbf{1} \cdot \mathbf{M}^{-\mathbf{1}}\right)}\right.$ \\
\hline WT & $0.64 \pm 0.03$ & $0.65 \pm 0.01$ & $1.0 \times 10^{3}$ \\
\hline D57A & $5.6 \pm 0.7$ & $0.025 \pm 0.001$ & 4.5 \\
D57N & $0.83 \pm 0.07$ & $0.021 \pm 0.0005$ & 25 \\
\hline S93A & $12 \pm 2$ & $0.045 \pm 0.003$ & 3.8 \\
S93N & $8.8 \pm 0.5$ & $0.057 \pm 0.001$ & 6.5 \\
\hline N128A & $26 \pm 3$ & $0.26 \pm 0.01$ & 10 \\
N128D & $21 \pm 2$ & $0.041 \pm 0.002$ & 2.0 \\
\hline
\end{tabular}

\subsection{D57, S93, and N128 Mutants Perturbed the Active Site Conformation in the Solution Structure}

NMR spectroscopy was used to identify the region associated with the DPN-triloop interaction in DUSP22. The backbone assignment of WT spectra was finished with $93 \%$ completion, and most of the residues in the D-loop, P-loop, and N-loop were detectable, including A56-S58, S60-L63, A90-R94, and N128-L135 (Figure 2A). The ${ }^{1} \mathrm{H}-{ }^{15} \mathrm{~N}-\mathrm{HSQC}$ spectra of S93N and N128A were collected to observe the perturbation. The spectra indicated that these mutants nearly perturbed the entire phosphatase domain of DUSP22. The D57, S93, and N128 signals disappeared, and the surrounding residues were perturbed in the structures (Figure S3). Unfortunately, S93N and N128A were difficult to assign because they were unstable in the triple resonance experiments. We used D57N to observe how the DPN-triloop interaction perturbed the structure in the solution conformation. The D57N mutant was stable, and the residues located in the D-loop, P-loop, and N-loop could be identified. Comparison of the ${ }^{1} \mathrm{H}-{ }^{15} \mathrm{~N}-\mathrm{HSQC}$ spectra of the WT and D57N showed that most backbone amide resonances in D57N were similar to those in the WT (Figure 2C), whereas the perturbed regions (more than $\Delta \delta+$ $2 \sigma$ ) were primarily located on the D-loop, P-loop, N-loop, and $\alpha$-helix $5(\alpha 5)$ (Figure 3A,B). The D57N mutation not only obviously changed the D-loop itself but also significantly perturbed the residues in the P-loop and N-loop.

The result was a local perturbation, revealing that the three loops can interact and perturb each other in solution conformation. The P-loop is a critical binding structure that provides the backbone amide and the side chain of arginine to interact with the phosphate groups of substrates (Figure 3C) [1,11]. In the perturbed region, we observed that the P-loop residues, G91, V92, and R94, were shifted in the D57N spectra and disappeared in the S93N and N128A spectra (Figures 2B and 3D-F). This revealed the conformational change in the P-loop, which probably affected the binding effect to reduce phosphatase activity. Moreover, the highest chemical shift perturbation (CSP) value was located on the N-loop motif, which perturbed the connected $\alpha$-helix, $\alpha 5$, and induced high CSP values (Figure 3B), suggesting that the conformational changes in the D-loop, P-loop, and N-loop can affect the surrounding structure. In this light, the perturbations in S93N and N128A mutants probably resulted from the conformational changes due to the disruption of the DPN-triloop interaction, indicating that more residues were perturbed by the mutations in solution structure (Figure 2B and Figure S3). These results revealed that D57, S93, and N128 contributed to forming the conformation of each loop and stabilized the structure of the active site. 
(A)



(B)

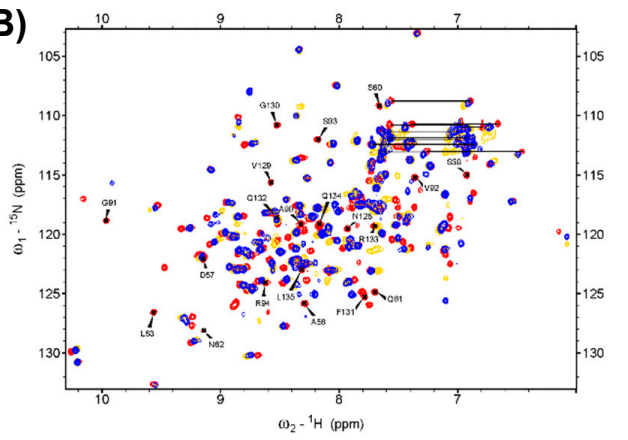

(C)

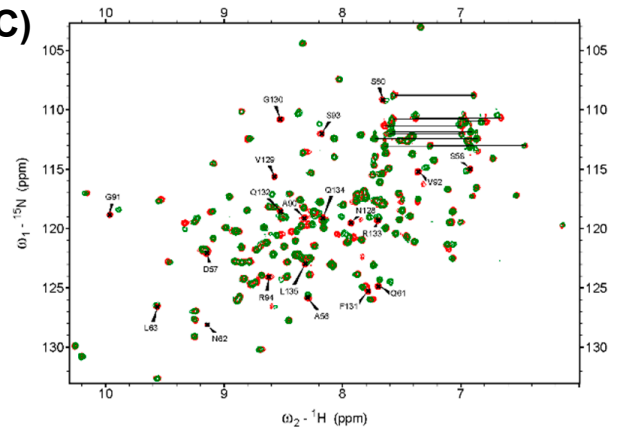

Figure 2. The backbone assignment of DUSP22_WT and the superimposed ${ }^{1} \mathrm{H}-{ }^{15} \mathrm{~N}-\mathrm{HSQC}$ spectra of the WT, S93N, N128A, and D57N. (A) The backbone amide resonance of residues in the WT is assigned on the ${ }^{1} \mathrm{H}-{ }^{15} \mathrm{~N}-\mathrm{HSQC}$ spectrum. It exhibited 139 backbone amide resonances of 150 expected resonances (5 prolines removed) in DUSP22 and showed $93 \%$ completion. (B) The ${ }^{1} \mathrm{H}-,{ }^{15} \mathrm{~N}-\mathrm{HSQC}$ spectrum of the WT (red) was superimposed with the spectra of S93N (yellow) and N128A (blue). The spectra indicated that the mutants critically perturbed the solution structures and shifted several residues. (C) The ${ }^{1} \mathrm{H}-{ }^{15} \mathrm{~N}-\mathrm{HSQC}$ spectrum of the WT (red) was superimposed with the spectra of D57N (green), which revealed the region perturbed by the DPN-triloop interaction. The labeled amino acids on the spectra were the residues in the D-loop, P-loop, and N-loop of the WT.

\subsection{The Structural Role of the DPN-triloop Interaction in Crystal Structures}

The crystal structures of C88S, C88S/S93A, C88S/S93N, WT_VO $\mathrm{VO}_{4}, \mathrm{~N} 128 \mathrm{~A}$, and N128D were determined in this study. All of the structures contained a ligand, $\mathrm{PO}_{4}, \mathrm{VO}_{4}$, or $\mathrm{SO}_{4}$, bound to the $\mathrm{P}$-loop in the active site. The structures of $\mathrm{WT}_{-} \mathrm{VO}_{4}$ and $\mathrm{C} 88 \mathrm{~S}_{-} \mathrm{PO}_{4}$ were similar, and their ligands were different from the previously published structures (PDB ID: 1WRM and 4WOH), which bound pTyr-like ligands $[17,28]$. The binding of the pTyr-like ligand was representative of the first step in the catalytic reaction, and the binding of vanadate represented the second step (Figure S1) [7]. The two structures were similar. Unlike Q262 in PTP1B, N128 did not rotate to stabilize water during the two catalytic steps (Figure S4). The DPN-triloop interaction was maintained in the catalytic reaction, suggesting that the stable interaction among the three loops was necessary for catalysis.

The S93 mutants were combined with a second mutation, C88S, to produce proteins for growing better crystals, which improved the resolution $(<1.6 \AA)$ and helped to determine the structures. 
The crystal structure of C88S was prepared to compare with the double mutants, and it formed the DPN-triloop interaction, which was similar to the WT (Figure 4A). In the crystal structure of C88S/S93A, the overall structure was similar to that of C88S, and the side chains of D57 and N128 also formed a hydrogen bond (Figure 4B). A water-mediated hydrogen bonding network was formed among the side chains of D57 and N128, as well as the backbone amide of N128. The position of this water molecule was above the original position of $\mathrm{S} 93$ and it did not form a hydrogen bond with the P-loop. A93 in the structure was independent of the water-mediated hydrogen bonding network, and the comparison of B-factor between C88S and C88S/S93A showed that the positional disorder increased on the three loops (Figure S5). The S93A mutation probably resulted in the unstable active site structure and decreased the catalytic efficiency. In contrast, the N93 in the crystal structure of C88S/S93N had a different effect and formed the hydrogen bonding with the side chain of D57 and the backbone amide of N128 (Figure 4C). The side chain of N128 also interacted with the side chain of D57. The DPN-triloop interaction formed but was not precise. This DPN-triloop interaction lacked a hydrogen bond between the side chains of $\mathrm{N} 93$ and N128, and the positions of D57 and N128 were shifted due to a spatial barrier from N93. The decrease in catalytic efficiency in S93N was associated with this conformational change. This result indicated that the formation of the DPN-triloop interaction through 593 induced precise spatial localization to stabilize active site structure and maintain phosphatase activity.

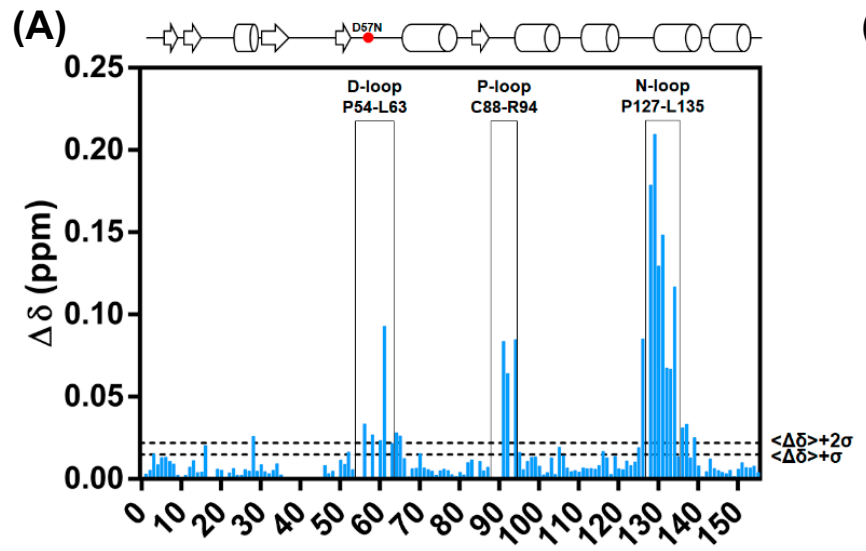

Residue Number

(C)



(D)

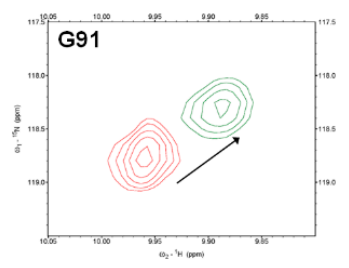

(B)

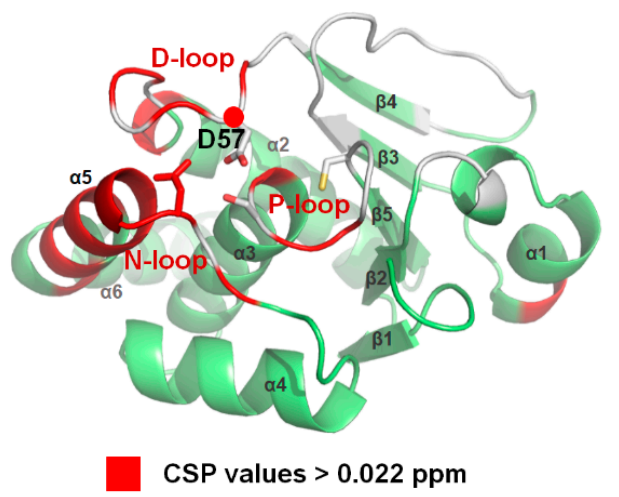

(F)

(E)



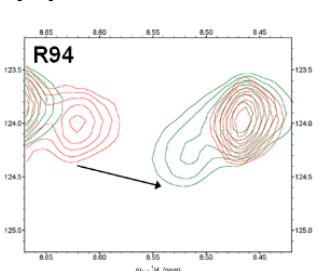

Figure 3. The D57N mutant exhibited a perturbed conformation as a result of DPN-triloop interactions. (A) The catalytic residue, D57, was replaced with asparagine, and the chemical shift perturbation (CSP) values were calculated. The threshold was set at $\Delta \delta+2 \sigma$, and the value was $0.022 \mathrm{ppm}$. The data indicated that the residues in the D-loop, P-loop, and N-loop were specifically perturbed by the D57N mutant. (B) The residues with CSP values greater than $\Delta \delta+2 \sigma$ are labeled on the DUSP22 structure in red. These results revealed the region that was perturbed by DPN-triloop interaction, including the D-loop, P-loop, N-loop, and $\alpha$-helix $5(\alpha 5)$. The spaces and white residues were non-assigned residues in the backbone assignment, including proline and disappeared signals in the 3D spectra. (C-F) The P-loop is the binding site, and the residues in the P-loop share their backbone amides as well as side chains to interact with the ligands. The ${ }^{1} \mathrm{H}-,{ }^{15} \mathrm{~N}-\mathrm{HSQC}$ spectrum of the WT (red) was superimposed with the spectrum of D57N (green) to show that the P-loop residues, G91, V92, and R94, were perturbed by disruption of the DPN-triloop interaction. 


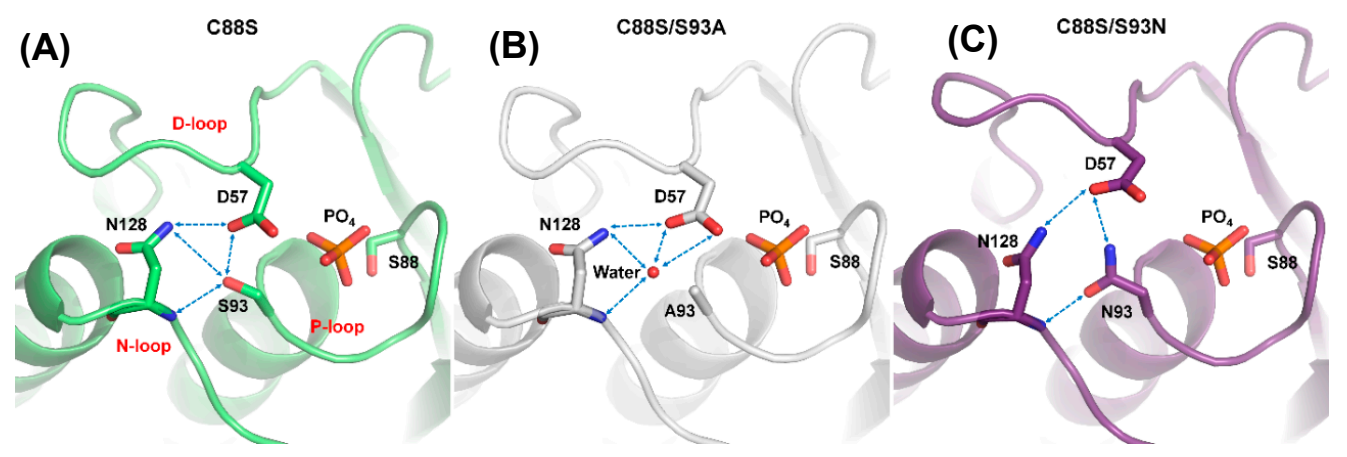

Figure 4. Disruption of DPN-triloop interaction in the crystal structures of S93 mutants. (A-C) The crystal structures of C88S (green), C88S/S93A (white), and C88S/S93N (purple) indicated how the mutation affected the hydrogen bonding network. The hydrogen bonds are shown by blue dotted lines, and a water molecule is shown as a red ball. (A) The crystal structure of C88S was prepared to compare with the S93 mutants, and the DPN-triloop interaction was unchanged. (B) S93 was replaced with alanine, and the structure was similar to that of C88S. A water molecule (W61) participated in forming the hydrogen bonding with the side chain of D57, the side chain of N128, and the backbone amide of N128. (C) S93 was replaced with a somatic mutant, asparagine. N93 formed the hydrogen bonding with the side chain of D57 and the backbone amide of N128.

Structural alignment among N128A, N128D, and WT_VO $\mathrm{VO}_{4}$ revealed that N128 participated in forming the conformation of the D-loop (Figure 5). In the N128A structure, one hydrogen bond was formed between the side chain of S93 and the backbone amide of A128. The interaction between D57 and N128 was broken, and no water-mediated hydrogen bonds connected them (Figure 5B). The D-loop was shifted away in the N128A mutant, indicating that the hydrogen bond from S93 was not sufficient to stabilize the D-loop. A similar result was observed in the N128D structure. The negative charge from D128 in the N128D mutant induced charge repulsion against D57, which resulted in unclear electron density in the conformation of the D-loop and slightly shifted the N-loop (Figure 5C). These results clearly indicated that the N128-D57 interaction anchored the D-loop and stabilized the conformation of the active site.

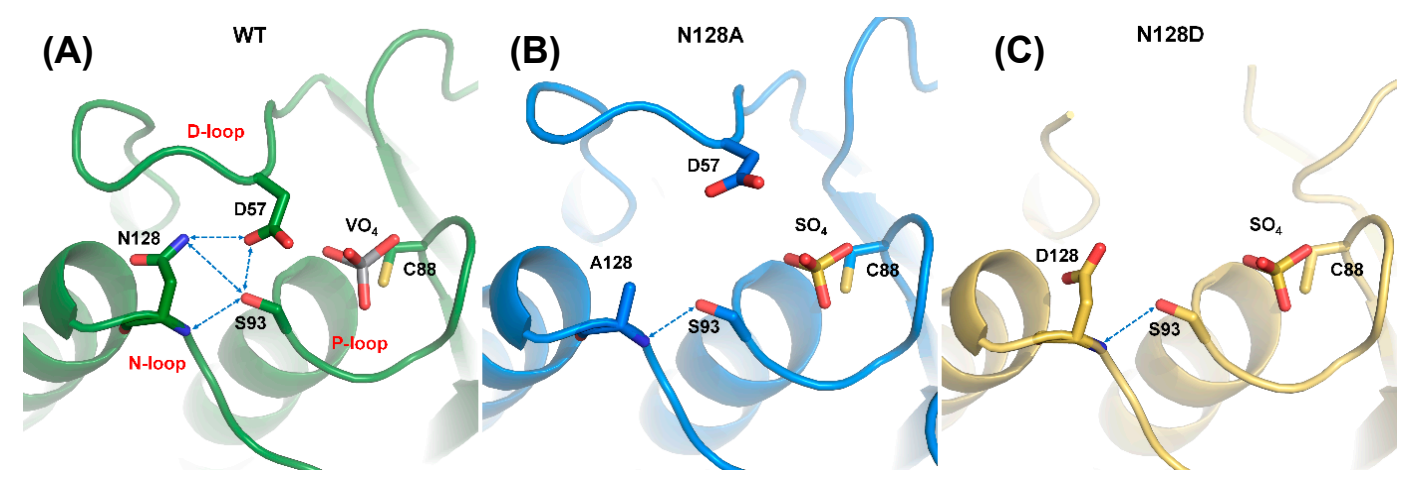

Figure 5. Disruption of DPN-triloop interaction in the crystal structures of N128 mutants. (A-C) The crystal structures of WT (green), N128A (blue), and N128D (yellow) indicated how the mutation affected the hydrogen bonding network and D-loop. The hydrogen bonds are shown by blue dotted lines. (A) The crystal structure of WT was co-crystallized with vanadate, and the DPN-triloop interaction was maintained. (B) N128 was replaced with alanine, and the conformation of the D-loop changed. Only one hydrogen bond was formed between the side chain of 593 and backbone amide of N128. (C) N128 was replaced with a somatic mutant, aspartate. The D128 induced charge repulsion with D57 and resulted in a dynamic D-loop that had unclear electron density. 


\section{Discussion}

N-loop-containing PTPs are the second largest family among Cys-based PTPs, and the characteristic of this family is formation of the DPN-triloop interaction in the active site. This conformation is crucial to maintain the phosphatase activity in DUSP22, and the forming mechanism is different from the water-mediated DPQ-triloop interaction in Q-loop-containing PTPs (Figure 1).

The structural difference between DPN-triloop interaction and DPQ-triloop interaction is probably associated with the specificity of the selection of ligands. These two groups have a common ancestral gene and divided during evolution [13]. N-loop-containing PTPs have a shallow and concise active site structure to interact with pSer, pThr, and pTyr, while Q-loop-containing PTPs extend the space of the active site pocket to specifically select pTyr. The purpose of the hydrogen bonding network in active sites is similar between DPN-triloop interaction and DPQ-triloop interaction. It positions the active site residues at a precise site for substrate interaction and catalysis. Our experiments provide evidence showing that completely forming the DPN-triloop interaction contributes to stabilizing the conformation of the active site.

The D-loop and P-loop need to be stabilized in the active site during the catalytic reaction. The DPN-triloop interaction stabilizes them and makes the three loops become a configuration. This means that the conformational change on each loop can perturb the others and change the conformation of the active site. Perturbation of the backbone amide in the P-loop is observed in the solution structure by disrupting the DPN-triloop interaction (Figures 2 and 3), which probably interferes with ligand binding as well as catalysis, and the perturbed conformation in S93A and S93N decreases the catalytic efficiency by 150-260 times (Table 1). In the crystal structure, the S93A mutant shows that the P-loop is independent from the interaction of the hydrogen bonding network and the B-factor is increase in the three loops (Figure 4B and Figure S5), suggesting that the conformation of the D-loop, P-loop, and N-loop cannot be stabilized. Even though S93N interacts with N128 and D57 in the crystal structure, the positions of D57 and N128 are different from those in the WT (Figure 4C), and the $\mathrm{N}$-loop and the D-loop are shifted. These results reveal that the conformation of the three loops is affected by the DPN-triloop interaction, and S93 contributes to the precise spatial position for forming hydrogen bonds. The larger side chains, asparagine, leucine, and phenylalanine (Table S1), are not suitable for this position, and these somatic mutations may interfere with the position of active site residues and abolish the phosphatase activity. Similar results are observed in N128 mutants. Disruption of the DPN-triloop interaction by N128A and N128D critically decrease the catalytic efficiency by 100-500 times (Table 1) because it induces conformation changes in the D-loop (Figure 5B-C) and perturbs the conformation of the P-loop (Figure 2B and Figure S3). The conformational change of the active site is the main reason for $K_{\mathrm{M}}$ increase, which is observed in most mutations. The $k_{\text {cat }}$ is associated with the interaction between D57 and S93, which can explain the difference between N128A and N128D. In the N128D structure, the charge repulsion induces D57 to be unable to interact with S93 and substrate, which decreases the $k_{\text {cat }}$. N128A does not form a barrier against D57, so D57 can interact with S93 due to D-loop dynamics and catalyze the substrate, while this interaction is not stable and induces a high $K_{\mathrm{M}}$. Consistently, the decrease in $k_{\text {cat }}$ is observed in the S93A mutant because D57 cannot form a stable interaction with S93. In the S93N mutant, N93 is a barrier that blocks D57 close to the substrate and decreases $k_{\text {cat }}$. The results of S93 and N128 verify that forming hydrogen bonds among D57, S93, and N128 are necessary to maintain the phosphatase activity. The DPN-triloop interaction stabilizes the residues in three loops at precise position and form the active site for facilitating the catalysis.

The residues in the region of the DPN-triloop interaction are potential sites for phosphatase activity regulation. Several somatic mutations are found in this region (Tables S1 and S2). Some of the mutations are on the critical residues, such as D57, C88, and R94, which directly abolish catalytic activity and disrupt substrate binding [29]. The mutation of S93F, deletion of N128, and equal mutations in other N-loop-containing PTPs (Table S1) $[15,16]$ can decrease the catalytic efficiency by disruption of the DPN-triloop interaction, and the mechanism is described in this study. Similarly, the mutations surrounded by D57, S93, and N128 might regulate phosphatase activity by changing the conformations 
of the D-loop, P-loop, and N-loop. In previous study, the allosteric sites in DUSP3 (VHR) and DUSP10 (MKP5) are reported, and these allosteric sites are surrounded by the N-loop and can affect the phosphatase activity $[30,31]$. Whether the mutations shown in Tables S1 and S2 participate in the process of cancer development is worth studying because N-loop-containing PTPs regulate several signaling pathways, and the deficiency or downregulation of these PTPs is frequently observed in cancer development [32,33]. Drug development to stabilize the DPN-triloop interaction is a possible strategy to benefit cancer treatment. However, some of the members participate in the promotion of cancer development, such as STYX, SSH1, and SSH3 [34-36]. Drug development requires more structural information for support, and our studies may serve as a foundation for further investigation of treatments focusing on N-loop-containing PTPs.

\section{Materials and Methods}

\subsection{Protein Expression and Purification}

The truncated DUSP22 (1-155 a.a.) was used in our experiments, and the purification method was modified from a previous study [17]. In brief, the truncated DUSP22 gene was ligated into pET21b (Merck KGaA, Darmstadt, Germany) with a hexa-histidine tag followed by a PreScission protease cleavage site (LEVLFQ/GP) on the N-terminus. Site-directed mutagenesis was performed by means of a KOD-plus Kit (Toyobo, Osaka, Japan). WT enzyme and most mutants were expressed by Rosetta 2 (DE3) (EMD Millipore, Billerica, MA, USA); meanwhile, the S93A mutant was expressed by BL21 (DE3) in a chaperone expression system (GroES and GroEL) (Takara Bio, Otsu, Shiga, Japan). The cells were grown at $37^{\circ} \mathrm{C}$ for $3 \mathrm{~h}$, and $1 \mathrm{mM}$ IPTG was added to induce protein expression at $25^{\circ} \mathrm{C}$ for $6-8 \mathrm{~h}$ or $16-20^{\circ} \mathrm{C}$ overnight. Cell pellets were resuspended in buffer A (20 mM PIPES (pH 7.0), $100 \mathrm{mM}$ $\mathrm{NaCl}$ ) with $1 \mathrm{mM}$ imidazole and then disrupted by sonication (Sonics \& Materials, Newtown, CT, USA). The supernatant was loaded onto a TALON metal affinity resin (Takara Bio) and eluted with buffer B (20 mM PIPES (pH 7.0), $100 \mathrm{mM} \mathrm{NaCl}, 150 \mathrm{mM}$ imidazole). The proteins were incubated with PreScission protease at $4{ }^{\circ} \mathrm{C}$ until complete cleavage of the His-tag, and loaded onto a size exclusion column (Sephacryl S-100 16/60) (GE Healthcare, Chicago, IL, USA) for elution by buffer A. The fractions were concentrated and stored at $-20^{\circ} \mathrm{C}$ until use.

\subsection{Kinetic Assay}

Phosphatase activity was detected by the cleavage of $p$-nitrophenyl phosphate (pNPP), and the method was modified from previous studies $[26,37,38]$. The kinetic assay was performed in reaction buffer (0.1 M sodium acetate, $0.05 \mathrm{M}$ Tris, and $0.05 \mathrm{M}$ Bis-Tris, $\mathrm{pH}$ 6.0). To calculate the $K_{\mathrm{M}}$ and $k_{\text {cat }}$ values, the protein concentration and substrate concentration were adjusted. WT and N128A were performed at a final concentration of $0.1 \mu \mathrm{M}$, and D57A, D57N, S93A, S93N, and N128D were performed at a final concentration of $1 \mu \mathrm{M}$. pNPP substrate was prepared for 11-12 concentrations in different ranges $(0.1-20 \mathrm{mM}$, or $1-50 \mathrm{mM}$ with or without $80 \mathrm{mM})$. Proteins and substrate were mixed in equal ratios and incubated at $30^{\circ} \mathrm{C}$ for $15 \mathrm{~min}$. The reaction was stopped by adding $2 \mathrm{~N} \mathrm{NaOH}$, and the absorbance at $405 \mathrm{~nm}$ was measured by NanoPhotometer Pearl (Implen, Westlake Village, CA, USA). The results were collected in triplicate experiments and fitted to the Michaelis-Menten equation in GraphPad Prism version 8 (GraphPad Software, San Diego, CA, USA).

\subsection{NMR Spectroscopy}

NMR spectra were collected on Bruker Avance II $600 \mathrm{MHz}$ and Bruker Avance III $850 \mathrm{MHz}$ spectrometers (Bruker BioSpin, Rheinstetten, Germany) at 298 K. For backbone assignment, ${ }^{15} \mathrm{~N}-,{ }^{13} \mathrm{C}$-labeled DUSP22_155WT was prepared at a final concentration of $1 \mathrm{mM}$ in NMR buffer (40 mM PIPES (pH 7.0), $100 \mathrm{mM} \mathrm{NaCl}, 10 \% \mathrm{D}_{2} \mathrm{O}(v / v)$ and $0.16 \mathrm{mM}$ DSS). The collected spectra included HSQC, HNCA, HN(CO)CA, HNCO, HNCACB, and HN(CO)CACB. The assignment of D57N was related to the WT spectra and confirmed by backbone assignment of the C $\alpha$ spectra 
( $\mathrm{HNCA}, \mathrm{HN}(\mathrm{CO}) \mathrm{CA})$ as well as ligand titration. For comparison of the mutants, ${ }^{15} \mathrm{~N}$-labeled proteins were prepared at the concentration of $0.1-0.2 \mathrm{mM}$ to collect ${ }^{1} \mathrm{H},{ }^{15} \mathrm{~N}$-heteronuclear single quantum coherence (HSQC) spectra. All spectra were processed by NMRpipe [39] and analyzed by Sparky [40]. The CSP values were calculated by the following formula:

$$
\operatorname{CSP}(p p m)=\sqrt{\frac{1}{2}\left(\delta_{H N}^{2}+\frac{\delta_{N}^{2}}{25}\right)}
$$

The threshold of CSPs was set at two standard deviations above the mean $(\Delta \delta+2 \sigma)$, which was calculated from $10 \%$ trimmed data $[30,41]$, and the residues with the CSP values above the threshold were labeled on the structure.

\subsection{Protein Crystallization}

Crystallization trials of $\mathrm{WT}_{-} \mathrm{VO}_{4}, \mathrm{C} 88 \mathrm{~S}, \mathrm{C} 88 \mathrm{~S} / \mathrm{S} 93 \mathrm{~A}, \mathrm{C} 88 \mathrm{~S} / \mathrm{S} 93 \mathrm{~N}, \mathrm{~N} 128 \mathrm{~A}$, and N128D were performed by hanging drop vapor diffusion. WT, C88S, N128A, and N128D were prepared in buffer A while C88S/S93A and C88S/S93N were prepared in buffer A with $2 \mathrm{mM} \mathrm{Li}_{2} \mathrm{SO}_{4}$ to prevent aggregation. The details of the protein concentrations and the reservoir solution are listed in Table S3. Proteins were mixed with reservoir solution at an equal ratio (1:1) and incubated at $20^{\circ} \mathrm{C}$. The WT crystal grew in $24 \mathrm{~h}$, and the crystal was soaked with a high concentration of $\mathrm{VO}_{4}$ in soaking solution (Table S3) for $48 \mathrm{~h}$. The crystals of C88S, C88S/S93A, and C88S/S93N grew in 16-24 h, and those of N128A and N128D grew in $48 \mathrm{~h}$.

\subsection{X-Ray Data Collection and Structure Determination}

The crystals were picked up and flash frozen in liquid nitrogen with 10-20\% glycerol. X-ray data were collected by TLS_15A, TLS_13B, and TPS_05A at NSRRC (Hsinchu, Taiwan). The collected data were processed by HKL2000 [42]. Crystal structures were obtained from Phaser-MR in Phenix [43], and the search model was WT DUSP22 (PDBID: 1WRM). The ligands, $\mathrm{VO}_{4}, \mathrm{PO}_{4}$, and $\mathrm{SO}_{4}$, were built and fit into crystal structures by eLBOW and LigandFit in Phenix. Refinement was performed and built manually in Phenix and Coot [44]. The data collection and refinement statistics are listed in Table S4. The protein structures of DUSP22_WT and the mutants were generated with PyMOL [45].

\subsection{Data Availability}

The backbone assignment of DUSP22_WT has been deposited in the Biological Magnetic Resonance Data Bank (BMRB ID: 50156). The atomic coordinates and structure factors of $\mathrm{WT}_{-} \mathrm{VO}_{4}, \mathrm{C} 88 \mathrm{~S}$, C88S/S93A, C88S/S93N, N128D, and N128A have been deposited in the Protein Data Bank (PDB ID codes 6LVQ, 6L1S, 6LMY, 6LOU, 6LOT, and 7C8S).

\section{Conclusions}

This study demonstrates the relationship between the DPN-triloop interaction and phosphatase activity in DUSP22. The DPN-triloop interaction forms the structure of the active site, and the mechanism differs from that of DPQ-triloop interaction. The DPN-triloop interaction stabilizes active site formation for substrate interaction and aligns the residues for catalysis. The high conservation of the D57 residue in the D-loop, the S93 residue in the P-loop, and the N128 residue in the N-loop explains the importance of the DPN-triloop interaction, which probably results in the active site formation in all N-loop-containing PTPs. Therefore, the DPN-triloop interaction may become a key for drug development to regulate the phosphatase activity of N-loop-containing PTPs. Our work provides a foundation for further study of active sites in different N-loop-containing PTPs and crucial information for investigating cancer mutations in the region of the DPN-triloop interaction. 
Supplementary Materials: Supplementary materials can be found at http://www.mdpi.com/1422-0067/21/20/ 7515/s1.

Author Contributions: Conceptualization, C.-H.L., H.-C.C. and P.-C.L.; investigation, C.-H.L. and C.-C.C.; validation, C.-H.L. and C.-C.C.; writing-original draft, C.-H.L.; writing-review and editing, P.-C.L.; resource, H.-C.C.; supervision, T.-H.T. and P.-C.L.; funding acquisition, H.-C.C., T.-H.T. and P.-C.L. All authors have read and agreed to the published version of the manuscript.

Funding: This work was supported by funding from the Ministry of Science and Technology (MOST), Taiwan (106-2311-B-007-004-MY3 to P.-C.L.; 107-2314-B-400-008 - and 107-2321-B-400-008 - to T.-H.T.; 107-2628-B-400-001 to H.-C.C.).

Acknowledgments: We thank the staff of the National Synchrotron Research Center (NSRRC) for their beamline preparation and technical support. We also thank Y.Z. Lee, C.Y. Chen, and the staff of the Instrumentation Center at National Tsing Hua University for assistance with the NMR experiments.

Conflicts of Interest: The authors declare no conflict of interest. The funders had no role in the design of the study; in the collection, analyses, or interpretation of data; in the writing of the manuscript, or in the decision to publish the results.

\section{Abbreviations}

$\begin{array}{ll}\text { Cys-based PTPs } & \text { Cysteine-based protein tyrosine phosphatases } \\ \text { DUSPs } & \text { Dual-specificity phosphatases } \\ \text { NMR } & \text { Nuclear magnetic resonance } \\ \text { P-loop } & \text { Phosphate binding loop }\end{array}$

\section{References}

1. Hobiger, K.; Friedrich, T. Voltage sensitive phosphatases: Emerging kinship to protein tyrosine phosphatases from structure-function research. Front. Pharmacol. 2015, 6, 20. [CrossRef] [PubMed]

2. Alonso, A.; Pulido, R. The extended human PTPome: A growing tyrosine phosphatase family. FEBS J. 2016, 283, 1404-1429. [CrossRef] [PubMed]

3. Patterson, K.I.; Brummer, T.; O’Brien, P.M.; Daly, R.J. Dual-specificity phosphatases: Critical regulators with diverse cellular targets. Biochem. J. 2009, 418, 475-489. [CrossRef] [PubMed]

4. Wang, Z.; Shen, D.; Parsons, D.W.; Bardelli, A.; Sager, J.; Szabo, S.; Ptak, J.; Silliman, N.; Peters, B.A.; Van der Heijden, M.S.; et al. Mutational analysis of the tyrosine phosphatome in colorectal cancers. Science 2004, 304, 1164-1166. [CrossRef]

5. Pramanik, K.; Chun, C.Z.; Garnaas, M.K.; Samant, G.V.; Li, K.; Horswill, M.A.; North, P.E.; Ramchandran, R. Dusp-5 and Snrk-1 coordinately function during vascular development and disease. Blood 2009, 113, 1184-1191. [CrossRef]

6. Zhao, S.; Sedwick, D.; Wang, Z. Genetic alterations of protein tyrosine phosphatases in human cancers. Oncogene 2015, 34, 3885-3894. [CrossRef]

7. Brandao, T.A.; Hengge, A.C.; Johnson, S.J. Insights into the reaction of protein-tyrosine phosphatase 1B: Crystal structures for transition state analogs of both catalytic steps. J. Biol. Chem. 2010, 285, 15874-15883. [CrossRef]

8. Pannifer, A.D.; Flint, A.J.; Tonks, N.K.; Barford, D. Visualization of the cysteinyl-phosphate intermediate of a protein-tyrosine phosphatase by x-ray crystallography. J. Biol. Chem. 1998, 273, 10454-10462. [CrossRef]

9. Zhao, Y.; Wu, L.; Noh, S.J.; Guan, K.L.; Zhang, Z.Y. Altering the nucleophile specificity of a protein-tyrosine phosphatase-catalyzed reaction. Probing the function of the invariant glutamine residues. J. Biol. Chem. 1998, 273, 5484-5492. [CrossRef]

10. Kuznetsov, V.I.; Hengge, A.C.; Johnson, S.J. New aspects of the phosphatase VHZ revealed by a high-resolution structure with vanadate and substrate screening. Biochemistry 2012, 51, 9869-9879. [CrossRef]

11. Yuvaniyama, J.; Denu, J.M.; Dixon, J.E.; Saper, M.A. Crystal structure of the dual specificity protein phosphatase VHR. Science 1996, 272, 1328-1331. [CrossRef] [PubMed]

12. Andersen, J.N.; Mortensen, O.H.; Peters, G.H.; Drake, P.G.; Iversen, L.F.; Olsen, O.H.; Jansen, P.G.; Andersen, H.S.; Tonks, N.K.; Moller, N.P. Structural and evolutionary relationships among protein tyrosine phosphatase domains. Mol. Cell. Biol. 2001, 21, 7117-7136. [CrossRef] [PubMed] 
13. Chen, M.J.; Dixon, J.E.; Manning, G. Genomics and evolution of protein phosphatases. Sci. Signal. $2017,10$. [CrossRef] [PubMed]

14. Jia, Z.; Barford, D.; Flint, A.J.; Tonks, N.K. Structural basis for phosphotyrosine peptide recognition by protein tyrosine phosphatase 1B. Science 1995, 268, 1754-1758. [CrossRef] [PubMed]

15. Tate, J.G.; Bamford, S.; Jubb, H.C.; Sondka, Z.; Beare, D.M.; Bindal, N.; Boutselakis, H.; Cole, C.G.; Creatore, C.; Dawson, E.; et al. COSMIC: The Catalogue of Somatic Mutations in Cancer. Nucleic Acids Res. 2019, 47, D941-D947. [CrossRef]

16. Zhang, J.; Bajari, R.; Andric, D.; Gerthoffert, F.; Lepsa, A.; Nahal-Bose, H.; Stein, L.D.; Ferretti, V. The International Cancer Genome Consortium Data Portal. Nat. Biotechnol. 2019, 37, 367-369. [CrossRef]

17. Yokota, T.; Nara, Y.; Kashima, A.; Matsubara, K.; Misawa, S.; Kato, R.; Sugio, S. Crystal structure of human dual specificity phosphatase, JNK stimulatory phosphatase-1, at 1.5 A resolution. Proteins 2007, 66, 272-278. [CrossRef]

18. Schwertassek, U.; Buckley, D.A.; Xu, C.F.; Lindsay, A.J.; McCaffrey, M.W.; Neubert, T.A.; Tonks, N.K. Myristoylation of the dual-specificity phosphatase c-JUN N-terminal kinase (JNK) stimulatory phosphatase 1 is necessary for its activation of JNK signaling and apoptosis. FEBS J. 2010, 277, 2463-2473. [CrossRef]

19. Li, J.P.; Yang, C.Y.; Chuang, H.C.; Lan, J.L.; Chen, D.Y.; Chen, Y.M.; Wang, X.; Chen, A.J.; Belmont, J.W.; Tan, T.H. The phosphatase JKAP/DUSP22 inhibits T-cell receptor signalling and autoimmunity by inactivating Lck. Nat. Commun. 2014, 5, 3618. [CrossRef]

20. Shen, Y.; Luche, R.; Wei, B.; Gordon, M.L.; Diltz, C.D.; Tonks, N.K. Activation of the Jnk signaling pathway by a dual-specificity phosphatase, JSP-1. Proc. Natl. Acad. Sci. USA 2001, 98, 13613-13618. [CrossRef]

21. Chen, A.J.; Zhou, G.; Juan, T.; Colicos, S.M.; Cannon, J.P.; Cabriera-Hansen, M.; Meyer, C.F.; Jurecic, R.; Copeland, N.G.; Gilbert, D.J.; et al. The dual specificity JKAP specifically activates the c-Jun N-terminal kinase pathway. J. Biol. Chem. 2002, 277, 36592-36601. [CrossRef] [PubMed]

22. Li, J.P.; Fu, Y.N.; Chen, Y.R.; Tan, T.H. JNK pathway-associated phosphatase dephosphorylates focal adhesion kinase and suppresses cell migration. J. Biol. Chem. 2010, 285, 5472-5478. [CrossRef] [PubMed]

23. Chuang, H.C.; Chen, Y.M.; Hung, W.T.; Li, J.P.; Chen, D.Y.; Lan, J.L.; Tan, T.H. Downregulation of the phosphatase JKAP/DUSP22 in T cells as a potential new biomarker of systemic lupus erythematosus nephritis. Oncotarget 2016, 7, 57593-57605. [CrossRef] [PubMed]

24. Zhou, R.; Chang, Y.; Liu, J.; Chen, M.; Wang, H.; Huang, M.; Liu, S.; Wang, X.; Zhao, Q. JNK Pathway-Associated Phosphatase/DUSP22 Suppresses CD4(+) T-Cell Activation and Th1/Th17-Cell Differentiation and Negatively Correlates with Clinical Activity in Inflammatory Bowel Disease. Front. Immunol. 2017, 8, 781. [CrossRef]

25. Xie, L.; Zhang, Y.L.; Zhang, Z.Y. Design and characterization of an improved protein tyrosine phosphatase substrate-trapping mutant. Biochemistry 2002, 41, 4032-4039. [CrossRef]

26. Denu, J.M.; Lohse, D.L.; Vijayalakshmi, J.; Saper, M.A.; Dixon, J.E. Visualization of intermediate and transition-state structures in protein-tyrosine phosphatase catalysis. Proc. Natl. Acad. Sci. USA 1996, 93, 2493-2498. [CrossRef]

27. Alonso, A.; Burkhalter, S.; Sasin, J.; Tautz, L.; Bogetz, J.; Huynh, H.; Bremer, M.C.; Holsinger, L.J.; Godzik, A.; Mustelin, T. The minimal essential core of a cysteine-based protein-tyrosine phosphatase revealed by a novel 16-kDa VH1-like phosphatase, VHZ. J. Biol. Chem. 2004, 279, 35768-35774. [CrossRef]

28. Lountos, G.T.; Cherry, S.; Tropea, J.E.; Waugh, D.S. Structural analysis of human dual-specificity phosphatase 22 complexed with a phosphotyrosine-like substrate. Acta Crystallogr Sect. F 2015, 71, 199-205. [CrossRef]

29. Flint, A.J.; Tiganis, T.; Barford, D.; Tonks, N.K. Development of "substrate-trapping" mutants to identify physiological substrates of protein tyrosine phosphatases. Proc. Natl. Acad. Sci. USA 1997, 94, 1680-1685. [CrossRef]

30. Cui, D.S.; Beaumont, V.; Ginther, P.S.; Lipchock, J.M.; Loria, J.P. Leveraging Reciprocity to Identify and Characterize Unknown Allosteric Sites in Protein Tyrosine Phosphatases. J. Mol. Biol. 2017, 429, 2360-2372. [CrossRef]

31. Gannam, Z.T.K.; Min, K.; Shillingford, S.R.; Zhang, L.; Herrington, J.; Abriola, L.; Gareiss, P.C.; Pantouris, G.; Tzouvelekis, A.; Kaminski, N.; et al. An allosteric site on MKP5 reveals a strategy for small-molecule inhibition. Sci. Signal. 2020, 13. [CrossRef] [PubMed] 
32. Meeusen, B.; Janssens, V. Tumor suppressive protein phosphatases in human cancer: Emerging targets for therapeutic intervention and tumor stratification. Int. J. Biochem. Cell Biol. 2018, 96, 98-134. [CrossRef] [PubMed]

33. Chen, H.F.; Chuang, H.C.; Tan, T.H. Regulation of Dual-Specificity Phosphatase (DUSP) Ubiquitination and Protein Stability. Int. J. Mol. Sci. 2019, 20, 2668. [CrossRef] [PubMed]

34. He, D.; Ma, Z.; Fang, C.; Ding, J.; Yang, W.; Chen, P.; Huang, L.; Wang, C.; Yu, Y.; Yang, L.; et al. Pseudophosphatase STYX promotes tumor growth and metastasis by inhibiting FBXW7 function in colorectal cancer. Cancer Lett. 2019, 454, 53-65. [CrossRef] [PubMed]

35. Wang, Y.; Kuramitsu, Y.; Kitagawa, T.; Baron, B.; Yoshino, S.; Maehara, S.; Maehara, Y.; Oka, M.; Nakamura, K. Cofilin-phosphatase slingshot-1L (SSH1L) is over-expressed in pancreatic cancer (PC) and contributes to tumor cell migration. Cancer Lett. 2015, 360, 171-176. [CrossRef] [PubMed]

36. Hu, Y.H.; Lu, Y.X.; Zhang, Z.Y.; Zhang, J.M.; Zhang, W.J.; Zheng, L.; Lin, W.H.; Zhang, W.; Li, X.N. SSH3 facilitates colorectal cancer cell invasion and metastasis by affecting signaling cascades involving LIMK1/Rac1. Am. J. Cancer Res. 2019, 9, 1061-1073.

37. Lorenz, U. Protein tyrosine phosphatase assays. Curr. Protoc. Immunol. 2011, 93. [CrossRef]

38. Alonso, A.; Narisawa, S.; Bogetz, J.; Tautz, L.; Hadzic, R.; Huynh, H.; Williams, S.; Gjorloff-Wingren, A.; Bremer, M.C.; Holsinger, L.J.; et al. VHY, a novel myristoylated testis-restricted dual specificity protein phosphatase related to VHX. J. Biol. Chem. 2004, 279, 32586-32591. [CrossRef]

39. Delaglio, F.; Grzesiek, S.; Vuister, G.W.; Zhu, G.; Pfeifer, J.; Bax, A. NMRPipe: A multidimensional spectral processing system based on UNIX pipes. J. Biomol. NMR 1995, 6, 277-293. [CrossRef]

40. Lee, W.; Tonelli, M.; Markley, J.L. NMRFAM-SPARKY: Enhanced software for biomolecular NMR spectroscopy. Bioinformatics 2015, 31, 1325-1327. [CrossRef]

41. Van Ingen, H.; Bonvin, A.M. Information-driven modeling of large macromolecular assemblies using NMR data. J. Magn. Reson. 2014, 241, 103-114. [CrossRef] [PubMed]

42. Otwinowski, Z.; Minor, W. Processing of X-ray diffraction data collected in oscillation mode. Methods Enzymol. 1997, 276, 307-326. [PubMed]

43. Adams, P.D.; Afonine, P.V.; Bunkoczi, G.; Chen, V.B.; Davis, I.W.; Echols, N.; Headd, J.J.; Hung, L.W.; Kapral, G.J.; Grosse-Kunstleve, R.W.; et al. PHENIX: A comprehensive Python-based system for macromolecular structure solution. Acta Crystallogr. Sect. D 2010, 66, 213-221. [CrossRef] [PubMed]

44. Emsley, P.; Lohkamp, B.; Scott, W.G.; Cowtan, K. Features and development of Coot. Acta Crystallogr Sect. D 2010, 66, 486-501. [CrossRef] [PubMed]

45. The PyMOL Molecular Graphics System; Version 1.3; Schrödinger LLC: New York, NY, USA, 2011.

Publisher's Note: MDPI stays neutral with regard to jurisdictional claims in published maps and institutional affiliations.

(C) 2020 by the authors. Licensee MDPI, Basel, Switzerland. This article is an open access article distributed under the terms and conditions of the Creative Commons Attribution (CC BY) license (http://creativecommons.org/licenses/by/4.0/). 